\title{
A Survey of Indigenous Tropical Legumes of Puerto Rico ${ }^{1}$
}

\author{
H. D. Dubey, R. Woodbury, G. L. Spain, and Rita L. Rodriguez ${ }^{2}$
}

\section{INTRODUCTION}

Nitrogen is the principal limiting factor among the major plant nutrients in economic crop production in most cultivated regions of the Tropics. Use of legumes in the cropping system is a very important way to supply this element, especially in those areas of the tropical world where farmers cannot afford costly nitrogen fertilizer. Certain legumes have been included in crop rotation as a long established practice in the old world Tropics. This practice is not followed in the Neotropics presumably because information about indigenous tropical legumes is lacking for this region.

Central and South America are among the most promising areas for finding tropical pasture legumes useful for grazing and improvement of soil nitrogen according to Bryan (3). Research on legumes in the Neotropics is, however, still in its infancy. Bermúdez García (2) found species in the genera Centrosema, Desmanthus, Desmodium, Stylosanthes, and Zornia in the Cauca Valley in Colombia of potential interest. Dirven (5) noted that 5 percent of the natural grassland vegetation in the coastal region of Surinam consisted of Leguminosae, chiefly Desmodium triflorum. Semple (7) reported that legumes in the Costa Rican grasslands constitute 5 to 25 percent of the vegetation. Of 79 legume species examined by De Souza in Trinidad, only 64 were nodulated (4).

Research and selection of tropical legumes for better adaptation, palatability, improved forage, seed yield and protein content is needed badly. A survey of root nodulation and efficiency of nitrogen fixation among native legumes is thus a logical initial step to reveal the Rhizobium strains of highest nitrogen-fixing capacity. Then, by cross-inoculation technique, the most efficient Rhizobium/legume association hopefully can be established.

To this end, a survey of the Island of Puerto Rico was conducted for indigenous tropical legumes conceivably having potential value for agricultural purposes. The results of this survey are given in this paper with brief descriptions of the nodules and field observations pertaining to the plants, soils, and environmental conditions. This information is of pertinence to rhizobiologists and research agronomists in other tropical and subtropical areas, and especially to agricultural scientists in Puerto Rico.

1 Manuscript submitted to Editorial Board July 3, 1973.

- Associate Soil Microbiologist, Botanist, Associate Agronomist and Research Assistant, respectively, Agricultural Experiment Station, University of Puerto Rico, Mayagüez Campus, Río Piedras, P.R. 
TABLE 1.-Species of legumes found in Puerto Rico with nodule characteristics, habitat, and plant growth habit

\begin{tabular}{|c|c|c|c|}
\hline Legume host & Nodule characteristics & Habitat & Plant growth habit \\
\hline \multicolumn{4}{|l|}{$\begin{array}{l}\text { Sub-family Papiliono- } \\
\text { ideae }\end{array}$} \\
\hline $\begin{array}{l}\text { Abrus praecatorius } \\
\text { L. }\end{array}$ & $\begin{array}{l}\text { Occurrence scarce in dry season, singly on } \\
\text { fine roots; round to oval, brownish white; } \\
2-3 \mathrm{~mm} \text { in diameter. }\end{array}$ & $\begin{array}{l}\text { Wide distribution throughout } \\
\text { the Island in open scrubby } \\
\text { areas, common in woodlots; } \\
\text { prefers well drained soil, } \\
\text { acid pH (4.6) with 114-203 } \\
\text { cm rainfall. }\end{array}$ & $\begin{array}{l}\text { A deep-rooted vigorous vine, } \\
\text { spreading over shrubs and } \\
\text { trees about } 300 \mathrm{~cm} \text { long; } \\
\text { flowering and fruiting } \\
\text { heavily. }\end{array}$ \\
\hline $\begin{array}{l}\text { Aeschynomene amer- } \\
\quad \text { icana } L \text {. }\end{array}$ & $\begin{array}{l}\text { Occurrence prolific, roots virtually covered } \\
\text { with nodules, confined to principal } \\
\text { branches where it forms rows of nodules; } \\
\text { round; smooth surface; pink; } 1.5 \mathrm{~mm} \text { di- } \\
\text { ameter. }\end{array}$ & $\begin{array}{l}\text { Widespread in moist roadside } \\
\text { and swampy depressions } \\
\text { throughout the Island in } \\
\text { acid to alkaline soil. }\end{array}$ & $\begin{array}{l}\text { Vigorous herbaceous shrub, } \\
165 \mathrm{~cm} \text { high; flowers and } \\
\text { fruits heavily. }\end{array}$ \\
\hline $\begin{array}{l}\text { Aeschynomene sensi- } \\
\text { tiva Sw. }\end{array}$ & $\begin{array}{l}\text { Occurrence rare, firmly attached to the } \\
\text { main roots; round; brownish white; } 1.5-2 \\
\text { mm diameter. }\end{array}$ & Same as $A$. americana $\mathrm{L}$. & Same as $A$. americana $\mathrm{L}$. \\
\hline $\begin{array}{l}\text { *Aeschynomene por- } \\
\text { toricensis Urban }\end{array}$ & $\begin{array}{l}\text { Occurs in fair numbers, singly distributed } \\
\text { on the main branches; round; white to } \\
\text { earthy grey; smooth surface; } 1-3 \mathrm{~mm} \text { in } \\
\text { diameter. }\end{array}$ & $\begin{array}{l}\text { Silicaceous, shale-derived, } \\
\text { moist, acid ( } \mathrm{pH} \text { 5.1) sandy } \\
\text { soil, high in organic matter. }\end{array}$ & $\begin{array}{l}\text { Rarely found, sparse grow- } \\
\text { ing, vine-like, about } 80 \mathrm{~cm} \\
\text { long, creeping; flowers and } \\
\text { fruits poorly. }\end{array}$ \\
\hline Cassia diffusa DC. & $\begin{array}{l}\text { Nodules few, scattered over the branch } \\
\text { roots; fairly firm texture; flattish, finger } \\
\text { or triangular shape; } 1-6 \mathrm{~mm} \text { long, ma- } \\
\text { jority } 4 \mathrm{~mm} \text {. }\end{array}$ & $\begin{array}{l}\text { A local plant, in silicaceous } \\
\text { sand. }\end{array}$ & $\begin{array}{l}\text { Diffuse, slender, deep rooted } \\
\text { plant; poor seeder. }\end{array}$ \\
\hline $\begin{array}{l}\text { *Cassia swartzii } \\
\text { Wickstr. }\end{array}$ & $\begin{array}{l}\text { Abundant, distributed over the whole root } \\
\text { system, firmly attached; polymorphic; } \\
\text { brownish red with white tips; 1-7 mm } \\
\text { long, majority } 5 \mathrm{~mm} \text {. }\end{array}$ & $\begin{array}{l}\text { Moist slope from low to higher } \\
\text { elevations; wide distribu- } \\
\text { tion in } 150-250 \mathrm{~cm} \text { rainfall } \\
\text { areas in all types of soils. }\end{array}$ & $\begin{array}{l}\text { Diffuse to erect shrub, } 60 \text { to } \\
180 \mathrm{~cm} \text { tall; fair seeder; or- } \\
\text { namental yellow flowers. }\end{array}$ \\
\hline
\end{tabular}




\begin{tabular}{|c|c|c|c|}
\hline $\begin{array}{l}\text { Sub-family Mimo- } \\
\text { soideae }\end{array}$ & & & \\
\hline $\begin{array}{l}\text { Desmanthus depres- } \\
\text { sus H. \& B. }\end{array}$ & $\begin{array}{l}\text { Occurs in fair numbers, singly, on fine roots } \\
\text { only; young nodules round, but mature } \\
\text { ones dumbbell shaped with a constriction } \\
\text { in middle; whitish grey color; young nod- } \\
\text { ules: } 1.5 \mathrm{~mm} \text { in diameter; mature nodules } \\
1.5 \mathrm{~mm} \text { wide and } 2.5-4 \mathrm{~mm} \text { long. }\end{array}$ & $\begin{array}{l}\text { Coastal slopes, dry to moist } \\
\text { areas, near neutral to alka- } \\
\text { line soil. }\end{array}$ & $\begin{array}{l}\text { Low growing slender diffused } \\
\text { plant; fair seeder. }\end{array}$ \\
\hline $\begin{array}{l}\text { Alysicarpus vaginalis } \\
\text { (L.) DC. }\end{array}$ & $\begin{array}{l}\text { 1-2 minute nodules found only on main } \\
\text { roots of very young seedling, nodules ob- } \\
\text { served only occasionally on adventitious } \\
\text { roots from the creeping stems; nodules } \\
\text { round with smooth surface; } 1-1.5 \mathrm{~mm} \text { in } \\
\text { size. }\end{array}$ & $\begin{array}{l}\text { Widely distributed in moist } \\
\text { open weedy areas in all } \\
\text { types of soils. }\end{array}$ & $\begin{array}{l}\text { Prostrate, matted vine; fruit- } \\
\text { ing heavily. }\end{array}$ \\
\hline $\begin{array}{c}\text { *Cracca caribaea } \\
\text { (Jacq.) Benth. }\end{array}$ & $\begin{array}{l}\text { Occurrence scarce, on main branch roots; } \\
\text { round becoming elongate finger-like with } \\
\text { a slight constriction in the middle; white } \\
\text { when young but grey when old; firm tex- } \\
\text { ture; surface striate; } 1-4 \mathrm{~mm} \text { long. }\end{array}$ & $\begin{array}{l}\text { Grows in open to moist forest, } \\
\text { mostly along southern } \\
\text { slopes, in alkaline soils (pH } \\
\text { 8.2). }\end{array}$ & $\begin{array}{l}\text { Sparse-growing shrub up to } \\
180 \mathrm{~cm} \text { high, more com- } \\
\text { monly } 60-90 \mathrm{~cm} \text {; fruiting } \\
\text { habit fair to poor. }\end{array}$ \\
\hline $\begin{array}{l}\text { Canavalia marilima } \\
\text { (Aubl.) Thou. }\end{array}$ & $\begin{array}{l}\text { Occurs in fair numbers, distributed on } \\
\text { branch roots; young nodules round, ma- } \\
\text { ture nodules lobed; surface hard; color } \\
\text { white which turns dark with age; fairly } \\
\text { big in size, range from } 1.5 \text { to } 2 \mathrm{~cm} \text { in di- } \\
\text { ameter. }\end{array}$ & $\begin{array}{l}\text { Alkaline sandy beach soils } \\
\text { throughout the Island. }\end{array}$ & $\begin{array}{l}\text { A long creeping beach plant; } \\
\text { flowering and fruiting } \\
\text { heavily. }\end{array}$ \\
\hline $\begin{array}{l}\text { *Calopogonium or- } \\
\text { thocarpum Urban }\end{array}$ & $\begin{array}{l}\text { Very few, found on main and adventitious } \\
\text { roots arising from the nodes of the } \\
\text { creeper; surface smooth or sometimes } \\
\text { slightly rough; emits a strong ammoni- } \\
\text { acal odor when bruised; } 1-3 \text { mm in diam- } \\
\text { eter. }\end{array}$ & $\begin{array}{l}\text { Scarcely found plant, occurs } \\
\text { in open, silicaceous sand } \\
\text { and wet, acid, peaty areas } \\
\text { (pH } 5.0 \text { ). }\end{array}$ & $\begin{array}{l}\text { A short prostrate vine; flow- } \\
\text { ering and fruiting sparse. }\end{array}$ \\
\hline
\end{tabular}




\begin{tabular}{|c|c|c|c|}
\hline Legume host & Nodule characteristics & Habitat & Plant growth habit \\
\hline $\begin{array}{l}\text { Desmanthus virgatus } \\
\text { (L.) Willd. }\end{array}$ & $\begin{array}{l}\text { Occurrence plenty, borne on fine braches; } \\
\text { whitish grey color; smooth waxy surface; } \\
\text { in size these were the biggest nodules en- } \\
\text { countered during this investigation. In } \\
\text { the month of August in an acid sandy } \\
\text { soil on the sea shore, nodules of unusually } \\
\text { big size and varied shapes were observed } \\
\text { on this legume; the nodules were radially } \\
\text { branched giving rise to different shapes, } \\
\text { such as fan or coral; } 1-3 \text { cm in dimension. } \\
\text { However, nodules occurring at other lo- } \\
\text { cations were finger or dumbbell shaped } \\
\text { and } 1-4 \text { mm in size. }\end{array}$ & Same as $D$. depressus. & $\begin{array}{l}\text { Erect growth, up to } 180 \mathrm{~cm} \text {; } \\
\text { heavy seeder. }\end{array}$ \\
\hline $\begin{array}{l}\text { Centrosema pubescens } \\
\text { Benth. }\end{array}$ & $\begin{array}{l}\text { Profuse nodulation, spread all over the } \\
\text { roots occurring close together especially } \\
\text { on branch roots, few large nodules found } \\
\text { only on thick roots; round; white; surface } \\
\text { reticulate; size range } 1-7 \mathrm{~mm} \text {, majority } \\
\text { measured } 3 \mathrm{~mm} \text {. }\end{array}$ & $\begin{array}{l}\text { In moist to well-drained areas } \\
\text { throughout the Island in all } \\
\text { types of acidic to alkaline } \\
\text { soils. }\end{array}$ & $\begin{array}{l}\text { A twining slender vine; flow- } \\
\text { ering and fruiting through- } \\
\text { out the year. }\end{array}$ \\
\hline $\begin{array}{l}\text { *Centrosema virgin- } \\
\text { iana (var. latifolia) } \\
\text { (L.) Benth. }\end{array}$ & $\begin{array}{l}\text { Very scarce, borne near the crown on prin- } \\
\text { cipal branches; round; surface reticulate, } \\
\text { translucent wall, nodule content clear to } \\
\text { light brown; } 2-5 \mathrm{~mm} \text { in diameter, ma- } \\
\text { jority } 3 \mathrm{~mm} \text {. }\end{array}$ & $\begin{array}{l}\text { Same as } C \text {. pubescens Benth. } \\
\text { but also found in acid beach } \\
\text { soil. }\end{array}$ & Same as C. pubescens Benth. \\
\hline $\begin{array}{l}\text { *Clitoria laurifolia } \\
\text { Poir. }\end{array}$ & $\begin{array}{l}\text { Scarce, on very fine upper branches; round; } \\
\text { yellowish brown; surface rough; } 2-3 \mathrm{~mm} \\
\text { in diameter. }\end{array}$ & $\begin{array}{l}\text { Moist, acid and alkaline sand; } \\
\text { occurrence very localized. }\end{array}$ & $\begin{array}{l}\text { A sparse vine, up to } 240 \mathrm{~cm} \\
\text { long; seeds commonly de- } \\
\text { stroyed by insect larvae. }\end{array}$ \\
\hline $\begin{array}{l}\text { Clitoria rubiginosa } \\
\text { Juss. }\end{array}$ & $\begin{array}{l}\text { Occurrence fair, spread all over the fine } \\
\text { roots, attached loosely and dislodge } \\
\text { easily; round; white; surface reticulate; } \\
\text { size up to } 2 \mathrm{~mm} \text {. }\end{array}$ & Same as $C$. laurifolia Poir. & Same as C. laurifolia Poir. \\
\hline
\end{tabular}

cipal branches; round; surface reticulate, translucent wall, nodule content clear to light brown; 2-5 $\mathrm{mm}$ in diameter, $\mathrm{ma}$ jority $3 \mathrm{~mm}$. yellowish brown; surface rough; 2-3 mm in diameter.

roots, attached loosely and dislodge easily; round; white; surface reticulate; size up to $2 \mathrm{~mm}$. 


\begin{tabular}{|c|c|c|c|}
\hline Crotalaria incana L. & $\begin{array}{l}\text { Nodules few, only on very young seedlings; } \\
\text { finger-like, pink; } 2-4 \mathrm{~mm} \text {. }\end{array}$ & $\begin{array}{l}\text { An uncommon weedy plant } \\
\text { growing in disturbed areas } \\
\text { on the southern sea coast; } \\
\text { alkaline soil pH. }\end{array}$ & $\begin{array}{l}\text { A small herbaceous shrub; } \\
\text { flowering and fruiting fair, } \\
\text { but seeds attacked by lar- } \\
\text { vae. }\end{array}$ \\
\hline Crotalaria retusa $\mathrm{L}$. & $\begin{array}{l}\text { Nodules attached mainly to principal roots } \\
\text { near the crown, branched like fingers of } \\
\text { the palm; smooth surface; white; pink or } \\
\text { purple, nodule terminals darker; nodules } \\
\text { easily dislodged; size range } 2 \mathrm{~mm}-1 \mathrm{~cm} .\end{array}$ & $\begin{array}{l}\text { Widespread in moist areas on } \\
\text { the sea coast as well as at } \\
\text { higher elevations. }\end{array}$ & Same as C. incana $\mathrm{L}$. \\
\hline $\begin{array}{l}\text { Crotalaria striata } \\
\text { DC. }\end{array}$ & $\begin{array}{l}\text { Nodules blocky, flat, branched, and frilled, } \\
\text { occurring an inch apart on the roots; } \\
\text { young small nodules pinkish brown, older } \\
\text { ones white and smoky; size range } 0.6 \\
\text { mm-1 cm. }\end{array}$ & $\begin{array}{l}\text { Moist disturbed areas from } \\
\text { coastal to higher elevations. }\end{array}$ & $\begin{array}{l}\text { A weedy gregarious shrub, up } \\
\text { to } 200 \mathrm{~cm} \text { high; flowering } \\
\text { and fruiting heavily; seeds } \\
\text { attacked by insect larvae. }\end{array}$ \\
\hline $\begin{array}{l}\text { *Dalbergia monetaria } \\
\text { L.f. }\end{array}$ & $\begin{array}{l}\text { Nodules few, attached to main and sec- } \\
\text { ondary thick roots; hard and woody; } \\
\text { rough surface; color reddish brown turn- } \\
\text { ing dark when old; } 1.5 \text { to } 3 \mathrm{~mm} \text { long. }\end{array}$ & $\begin{array}{l}\text { A local widespread plant of } \\
\text { rocky ravines and open } \\
\text { woods of medium eleva- } \\
\text { tions ( } 300 \text { meters); soil quite } \\
\text { acid (pH } 4.7 \text { ). }\end{array}$ & $\begin{array}{l}\text { A large woody vine; up to } 900 \\
\text { cm long; flowering and } \\
\text { fruiting heavily. }\end{array}$ \\
\hline $\begin{array}{l}\text { Desmodium adscend- } \\
\text { ens (Sw.) DC. }\end{array}$ & $\begin{array}{l}\text { Profuse nodulation, occur along the prin- } \\
\text { cipal branches; round; pink; smooth sur- } \\
\text { face; } 1.5-2 \mathrm{~mm} \text { in size. }\end{array}$ & $\begin{array}{l}\text { Disturbed, moist to wet areas; } \\
\text { in low to high elevations; } \\
\text { acid soil of pH } 5 \text {. }\end{array}$ & $\begin{array}{l}\text { A procumbent plant growing } \\
\text { in mats, vigorous grower } \\
\text { and heavy seeder. }\end{array}$ \\
\hline $\begin{array}{l}\text { Desmodium axillare } \\
\text { (Sw.) DC. }\end{array}$ & $\begin{array}{l}\text { Profuse nodulation, nodules occur on the } \\
\text { adventitious roots arising from the nodes } \\
\text { of the creeper, occurring in clumps of } 2 \text { to } \\
4 \text { nodules at one place; uniformly round; } \\
\text { grey color; size } 2-5 \mathrm{~mm} \text {. }\end{array}$ & $\begin{array}{l}\text { In moist open woods, having } \\
125-150 \mathrm{~cm} \text { rainfall; slight]y } \\
\text { acid to neutral soils. }\end{array}$ & $\begin{array}{l}\text { A vigorous, matted, stolonif- } \\
\text { erous plant; seeding fairly } \\
\text { abundant. }\end{array}$ \\
\hline $\begin{array}{l}\text { Desmodium barbatum } \\
\text { (L.) Benth. }\end{array}$ & $\begin{array}{l}\text { Nodules abundant, mostly in the vicinity } \\
\text { of the main root, on principal branches; } \\
\text { round; smooth surface; brown; } 1.5 \mathrm{~mm} \\
\text { average size. }\end{array}$ & $\begin{array}{l}\text { Locally abundant, in moist } \\
\text { acid (pH 4.6) sandy soil. }\end{array}$ & $\begin{array}{l}\text { Annual herb, up to } 60 \mathrm{~cm} \\
\text { high; seeding profusely. }\end{array}$ \\
\hline
\end{tabular}




\begin{tabular}{|c|c|c|c|}
\hline Legume host & Nodule characteristics & Habitat & Plant growth habit \\
\hline $\begin{array}{l}\text { Desmodium canum } \\
\text { (gmel.) Schinz \& } \\
\text { Thellung }\end{array}$ & $\begin{array}{l}\text { Uniformly distributed, at the end of very } \\
\text { fine roots; round; brown; surface has a } \\
\text { fine prickly and cracked appearance; } 2-3 \\
\text { mm size. }\end{array}$ & $\begin{array}{l}\text { Disturbed areas and laws } \\
\text { throughout the Island; acid } \\
\text { (pH 4.8) to near neutral } \\
\text { soils. }\end{array}$ & $\begin{array}{l}\text { Prostrate to bushy, low } \\
\text { shrub; flowering and seed- } \\
\text { ing heavily. }\end{array}$ \\
\hline $\begin{array}{c}\text { Desmodium procum- } \\
\text { bens (Mill) Hitche. }\end{array}$ & $\begin{array}{l}\text { Nodules few attached to branching roots; } \\
\text { round; white or greenish when young, } \\
\text { turning pinkish brown when mature; sur- } \\
\text { face streaked; } 1-2.5 \mathrm{~mm} \text { in size. }\end{array}$ & $\begin{array}{l}\text { Found mostly in dry to moist } \\
\text { southern slopes, with } 760 \\
\text { cm rainfall; alkaline soil } \\
\text { (pH 7.5). }\end{array}$ & $\begin{array}{l}\text { A weak vine-like shrub; fair } \\
\text { seeder. }\end{array}$ \\
\hline $\begin{array}{l}\text { Desmodium molle } \\
\text { (Vahl.) DC. }\end{array}$ & $\begin{array}{l}\text { Nodules present in fair number on branch } \\
\text { roots; round when young but lobed and } \\
\text { streaked when mature; most nodules } \\
\text { earthy cream color, but some pink col- } \\
\text { ored; } 1-3.5 \mathrm{~mm} \text { in size. }\end{array}$ & Same as D. procumbens. & $\begin{array}{l}\text { A seasonal shrub up to } 180 \\
\text { cm high; flowering and } \\
\text { fruiting fair. }\end{array}$ \\
\hline $\begin{array}{l}\text { Desmodium pur- } \\
\text { pureum (Mill.) } \\
\text { Fawc, \& Rendle }\end{array}$ & $\begin{array}{l}\text { Occurs in fair number, singly on very fine } \\
\text { branches; round; grey; surface rough } \\
\text { showing streaks; easily dislodged; size } \\
1.5-4 \mathrm{~mm} \text {. }\end{array}$ & $\begin{array}{l}\text { Same as } D . \text { molle and } D . \text { pro- } \\
\text { cumbens. }\end{array}$ & $\begin{array}{l}\text { Vigorous shrub up to } 240 \mathrm{~cm} \\
\text { tall; flowering and fruiting } \\
\text { heavily. }\end{array}$ \\
\hline $\begin{array}{l}\text { *Desmodium sin- } \\
\text { tenisii Urban }\end{array}$ & $\begin{array}{l}\text { Very few, borne on fine lateral branches; } \\
\text { round; dark grey; uniformly rough sur- } \\
\text { face with whitish fissures; } 1 \text { to } 3 \mathrm{~mm} \text { di- } \\
\text { ameter, majority } 2 \mathrm{~mm} \text {. }\end{array}$ & $\begin{array}{l}\text { Rare local plant of moist lime- } \\
\text { stone hills. }\end{array}$ & $\begin{array}{l}\text { Prostrate stoliniferous plant; } \\
\text { fruiting light. }\end{array}$ \\
\hline $\begin{array}{l}\text { Desmodium scorpi- } \\
\text { urus (Sw.) Kuntze }\end{array}$ & $\begin{array}{l}\text { Borne on thick main roots, most abundant } \\
\text { near the crown; round; pinkish brown; } \\
\text { smooth surface with whitish cracks; } 1-3 \\
\text { mm. }\end{array}$ & $\begin{array}{l}\text { Infrequent but wide spread } \\
\text { in moist areas; soil } \mathrm{pH} \text { near } \\
8 .\end{array}$ & $\begin{array}{l}\text { Vigorous, matted, low shrub; } \\
\text { heavy seeder. }\end{array}$ \\
\hline $\begin{array}{l}\text { Desmodium triflorum } \\
\text { (L.) DC. }\end{array}$ & $\begin{array}{l}\text { Occurs in fair numbers, attached firmly to } \\
\text { main roots of the creeper; round; white, } \\
\text { brown or pink colored; } 1.5-2 \mathrm{~mm} \text { diame- } \\
\text { ter. }\end{array}$ & $\begin{array}{l}\text { Common to moist roadsides } \\
\text { and disturbed areas, in low } \\
\text { to middle elevations; soil } \\
\text { pH may vary from acid (5.1) } \\
\text { to alkaline (8.2). }\end{array}$ & $\begin{array}{l}\text { A matted carpet-like growth; } \\
\text { less than } 5 \mathrm{~cm} \text { high; flower- } \\
\text { ing and seeding fair. }\end{array}$ \\
\hline
\end{tabular}
$\mathrm{pH}$ may vary from acid (5.1) to alkaline (8.2). 


\begin{tabular}{|c|c|c|c|}
\hline Galactia dubia DC. & $\begin{array}{l}\text { Very scarce, on well developed white root } \\
\text { and its branches; young nodules round } \\
\text { but older ones of irregular shapes mostly } \\
\text { finger-like and branching; light yellow } \\
\text { turning brown with age; } 2 \text { to } 4 \mathrm{~mm} \text { long, } \\
\text { sometimes as large as } 12 \times 6 \mathrm{~mm} \text {. }\end{array}$ & $\begin{array}{l}\text { Infrequent, in coastal open } \\
\text { areas and dry woodlands; } \\
\text { alkaline soils. }\end{array}$ & $\begin{array}{l}\text { A slender vine up to } 240 \mathrm{~cm} \\
\text { long twining on shrubs; } \\
\text { fruiting fair. }\end{array}$ \\
\hline $\begin{array}{l}\text { Galactia striata } \\
\text { (Jacq.) Urban }\end{array}$ & $\begin{array}{l}\text { Extremely small nodules distributed all } \\
\text { over the fine root system, occasionally } \\
2-3 \text { bigger nodules present; round; white; } \\
\text { bigger nodules measure } 1.5-4 \mathrm{~mm} \text { in di- } \\
\text { ameter. }\end{array}$ & Same as $G$. dubia DC. & $\begin{array}{l}\text { Same as } G \text {. dubia but heavy } \\
\text { seeder. }\end{array}$ \\
\hline $\begin{array}{l}\text { Indigofera suffructi- } \\
\text { cosa Mill. }\end{array}$ & $\begin{array}{l}\text { Few nodules present on very fine branches; } \\
\text { polymorphic in shape (round, oval, finger- } \\
\text { like, cuboid), color varied e.g., white, } \\
\text { yellowish or brown; size range 1-6 mm. }\end{array}$ & $\begin{array}{l}\text { Dry to moist open areas from } \\
\text { cost to middle elevations; } \\
\text { soil pH near neutral. }\end{array}$ & $\begin{array}{l}\text { A suffrutescent greyish } \\
\text { shrub, up to } 240 \mathrm{~cm} \text { tall; } \\
\text { flowering and fruiting } \\
\text { heavy. }\end{array}$ \\
\hline $\begin{array}{l}\text { Phaseolus adenan- } \\
\text { thus G. F. W. } \\
\text { Meyer }\end{array}$ & $\begin{array}{l}\text { Nodules plenty, distributed all over the } \\
\text { root system; round; greyish with white } \\
\text { streaks; rough surface; } 0.5-4 \mathrm{~mm} \text { in size. }\end{array}$ & $\begin{array}{l}\text { Moist woodlots and moist } \\
\text { open forest throughout the } \\
\text { Island; wide range of soil } \\
\text { pH from acid to alkaline. }\end{array}$ & $\begin{array}{l}\text { Weedy twining vine up to } 600 \\
\text { cm long; seeding fair. }\end{array}$ \\
\hline $\begin{array}{l}\text { Phaseolus lathyroides } \\
\text { L. }\end{array}$ & $\begin{array}{l}\text { Profuse nodulation, clustered around the } \\
\text { main roots near the crown; round; pink } \\
\text { nodules found only in March-May; old } \\
\text { nodules blue to dark brown; surface stri- } \\
\text { ated; most nodules measure } 2.5-3 \mathrm{~mm} \text {, } \\
\text { but a few to } 6-7 \mathrm{~mm} \text {. }\end{array}$ & $\begin{array}{l}\text { Most commonly seen legume } \\
\text { in the Island; found in } \\
\text { fields, roadsides, and dis- } \\
\text { turbed sites through the } \\
\text { Island under a wide range } \\
\text { of soil pH. }\end{array}$ & $\begin{array}{l}\text { Herbaceous shrub or vine- } \\
\text { like, up to } 180 \mathrm{~cm} \text {, normally } \\
90-180 \mathrm{~cm} \text { tall; flowering } \\
\text { and fruiting heavily. }\end{array}$ \\
\hline $\begin{array}{l}\text { *Phaseolus tricho- } \\
\text { carpus C. Wright }\end{array}$ & $\begin{array}{l}\text { Nodules very scarce, borne on branch and } \\
\text { adventitious roots growing from stem } \\
\text { nodes; round; surface smooth; pinkish } \\
\text { green color with white cracks; } 1 \text { to } 2 \mathrm{~mm} \text {. }\end{array}$ & $\begin{array}{l}\text { In moist acid sandy peat ( } \mathrm{pH} \\
5.1 \text { ); occurrence very rare. }\end{array}$ & $\begin{array}{l}\text { Herbaceous slender vine } \\
\text { rooting at nodes. }\end{array}$ \\
\hline $\begin{array}{l}\text { *Vigna hosei } \\
\text { (Craib.) Back }\end{array}$ & $\begin{array}{l}\text { Occurs in sufficient numbers principally on } \\
\text { thick main roots, not easily dislodged; } \\
\text { round but tend to form } 3 \text { to } 4 \text { lobes; pink } \\
\text { color; smooth surface; } 1-3 \text { mm diameter, } \\
\text { usually } 2 \text { mm. }\end{array}$ & $\begin{array}{l}\text { Locally abundant in moist } \\
\text { northern slopes; in near } \\
\text { neutral soils. }\end{array}$ & $\begin{array}{l}\text { A slender, matted, stolonif- } \\
\text { erous vine, sparse fruiting. }\end{array}$ \\
\hline
\end{tabular}


TABLE 1.-Continued

\begin{tabular}{|c|c|c|c|}
\hline Legume host & Nodule characteristics & Habitat & Plant growth habit \\
\hline $\begin{array}{l}\text { Rhynchosia minima } \\
\text { (L.) DC. }\end{array}$ & $\begin{array}{l}\text { Occurrence scarce, distributed over whole } \\
\text { root system, borne singly; round or oval } \\
\text { on fine branches; long finger-like on main } \\
\text { roots; surface reticulate; color grey with } \\
\text { whitish cracks; size } 4-5 \mathrm{~mm} \text {. }\end{array}$ & $\begin{array}{l}\text { Abundant, from coastal to } \\
\text { middle elevations in well } \\
\text { drained soils, in southern } \\
\text { part of the Island having } \\
\text { alkaline soil. }\end{array}$ & $\begin{array}{l}\text { A vigorous slender vine, but } \\
\text { highly susceptible to leaf } \\
\text { mosaic; heavy fruiting. }\end{array}$ \\
\hline $\begin{array}{l}\text { *Rhynchosia reticu- } \\
\text { lata (Sw.) DC. }\end{array}$ & $\begin{array}{l}\text { Very scarce, only few plants found nodu- } \\
\text { lated with } 2 \text { to } 3 \text { nodules present per } \\
\text { plant, borne on very fine lateral roots; } \\
\text { easily dislodged; round; yellow; surface } \\
\text { rough; } 1.5-3 \mathrm{~mm} \text { in diameter, majority } 2 \\
\text { mm. }\end{array}$ & $\begin{array}{l}\text { Less abundant and found in } \\
\text { drier areas than } R \text {. minima; } \\
\text { alkaline soil } \mathrm{pH} \text {. }\end{array}$ & $\begin{array}{l}\text { A larger vine than } R \text {. minima; } \\
\text { heavy fruiting. }\end{array}$ \\
\hline $\begin{array}{l}\text { Sesbania sericea } \\
\text { (Willd.) DC. }\end{array}$ & $\begin{array}{l}\text { Occurrence abundant, in clusters or strings } \\
\text { on thick main branches mostly near } \\
\text { crown, oval or egg shape; some nodules } \\
\text { so close together that they coalesce giv- } \\
\text { ing rise to relatively big nodules; nodules } \\
\text { firmly attached; surface smooth; nodules } \\
\text { appear pink in June-July, and brown at } \\
\text { other times; size } 3-4 \mathrm{~mm} \text {. }\end{array}$ & $\begin{array}{l}\text { Heavy poorly drained marl } \\
\text { clay near the coast, in alka- } \\
\text { line clay soils derived from } \\
\text { shell. }\end{array}$ & $\begin{array}{l}\text { An annual shrub up to } 300 \mathrm{~cm} \\
\text { high; heavy seeder. }\end{array}$ \\
\hline $\begin{array}{l}\text { Stylosanthes hamiata } \\
\text { (L.) Taubert }\end{array}$ & $\begin{array}{l}\text { Small round nodules of uniform size occur- } \\
\text { ring singly on fairly thick branch roots. } \\
\text { The roots present an appearance of } \\
\text { spiked-fence wire with very short } \\
\text { branches arising in bunches at intervals. } \\
\text { Nodules mostly brown, some pink; } \\
\text { smooth surface; size } 1-1.5 \mathrm{~mm} \text {. }\end{array}$ & $\begin{array}{l}\text { Locally abundant in coastal } \\
\text { dry to moist open areas and } \\
\text { roadsides, with alkaline soil } \\
\text { pH. }\end{array}$ & $\begin{array}{l}\text { A sparse growing, low shrub; } \\
\text { seeding year-round. }\end{array}$ \\
\hline
\end{tabular}




\begin{tabular}{|c|c|c|c|}
\hline $\begin{array}{l}\text { *Tephrosia cathar- } \\
\text { tica (Sesse \& Moc.) } \\
\text { Urban }\end{array}$ & $\begin{array}{l}\text { Nodules few, scattered on fine branches } \\
\text { not borne terminally; mature nodules } \\
\text { finger-like, sometimes the long ones are } \\
\text { bifurcated; wall noticeably transparent } \\
\text { through which the inner darker content } \\
\text { is visible; very young nodules measured } \\
2 \mathrm{~mm} \text { in diameter, older finger-like nod- } \\
\text { ules were up to } 10 \times 3 \mathrm{~mm} \text {. }\end{array}$ & $\begin{array}{l}\text { Coastal alkaline sand and } \\
\text { drier areas. }\end{array}$ & $\begin{array}{l}\text { A low growing greyish annual } \\
\text { shrub; heavy seeder. }\end{array}$ \\
\hline $\begin{array}{l}\text { Tephrosia cineria } \\
\text { (L.) Pers. }\end{array}$ & $\begin{array}{l}\text { Occurrence fair, scattered along the fine } \\
\text { branches, not borne terminally; mostly } \\
\text { long finger-like, some bifurcate; wall al- } \\
\text { most transparent through which the dark } \\
\text { inside content visible; size ranged from } \\
2 \mathrm{~mm} \text { in diameter for round nodules to } \\
10 \times 3 \mathrm{~mm} \text { for long nodules. }\end{array}$ & $\begin{array}{l}\text { Open sea coasts to coastal } \\
\text { slopes with alkaline soil } \mathrm{pH} \text {. }\end{array}$ & $\begin{array}{l}\text { Vine-like, procumbent, grey } \\
\text { ish plants; heavy seeder. }\end{array}$ \\
\hline $\begin{array}{l}\text { Teramnus labialus } \\
\text { (L. f.) Spreng. }\end{array}$ & $\begin{array}{l}\text { Very few nodules, on very fine branches, } \\
\text { delicately attached so that they are easily } \\
\text { dislodged; round; surface reticulate and } \\
\text { transparent through which the inner dark } \\
\text { content visible; young nodules clear } \\
\text { white, older show dark content; size } \\
1-4 \mathrm{~mm} \text {. }\end{array}$ & $\begin{array}{l}\text { Coastal sand and shrubby } \\
\text { areas in the southern part } \\
\text { of the Island with alkaline } \\
\text { soil (pH 7.8). }\end{array}$ & $\begin{array}{l}\text { Slender twining vine; fair } \\
\text { seeder. }\end{array}$ \\
\hline $\begin{array}{l}\text { Teramnus uncinatus } \\
\text { (L.) Sw. }\end{array}$ & $\begin{array}{l}\text { Occurrence fair, borne singly all over the } \\
\text { root system; round; earthy grey; 1-2 } \mathrm{mm} \\
\text { in diameter. }\end{array}$ & $\begin{array}{l}\text { An uncommon vine found } \\
\text { mostly in thickets at lower } \\
\text { to middle elevations; soil } \\
\text { with near neutral pH. }\end{array}$ & $\begin{array}{l}\text { A slender hairy vine, } 180 \mathrm{~cm} \\
\text { long; flowering and seeding } \\
\text { fair. }\end{array}$ \\
\hline $\begin{array}{l}\text { Vigna repens (L.) } \\
\text { Kuntze }\end{array}$ & $\begin{array}{l}\text { Occurrence profuse during March and April } \\
\text { but scarce in December; when profuse } \\
\text { they occur all along the roots arising } \\
\text { from the creeping stem; occur in clumps } \\
\text { of } 4-5 \text { at intervals of } 10 \text { to } 25 \mathrm{~mm} \text { on thick }\end{array}$ & $\begin{array}{l}\text { Abundant in coastal wet } \\
\text { areas, also found at higher } \\
\text { elevations; very acid (pH } \\
\text { 4.5) to neutral soils. }\end{array}$ & $\begin{array}{l}\text { Herbaceous, creeping or } \\
\text { twining vine with orna } \\
\text { mental yellow flowers } \\
\text { heavy seeder. }\end{array}$ \\
\hline
\end{tabular}




\begin{tabular}{|c|c|c|c|}
\hline Legume host & Nodule characteristics & Habitat & Plant growth habit \\
\hline & $\begin{array}{l}\text { fleshy roots; also occur as beaded chain } \\
\text { along roots; round; smooth surface; pink } \\
\text { color; usual size of individual nodules } 3 \\
\text { mm diameter when in cluster but when } \\
\text { occurring isolated and singly they tend } \\
\text { to grow larger, } 4-5 \mathrm{~mm} \text { in diameter. }\end{array}$ & & \\
\hline $\begin{array}{l}\text { Vigna vexillata (L.) } \\
\text { A Rich. }\end{array}$ & $\begin{array}{l}\text { Occurrence scarce, attached mostly to sec- } \\
\text { ondary roots, a few on main roots, not } \\
\text { present on fine roots; very young nodules } \\
\text { are round, becoming } 3-4 \text { lobed as they } \\
\text { grow; surface becoming rough in fully } \\
\text { grown nodules; color white in young nod- } \\
\text { ules turning to pink with white streaks, } \\
\text { in mature nodules; size } 1-4 \text { mm in diame- } \\
\text { ter. }\end{array}$ & $\begin{array}{l}\text { A local plant of open or grassy } \\
\text { fields, in very acid soils ( } \mathrm{pH} \\
4.5 \text { ). }\end{array}$ & $\begin{array}{l}\text { A long creeping vine rooting } \\
\text { at the nodes; fair seeder. }\end{array}$ \\
\hline $\begin{array}{l}\text { Zornia diphylla (L.) } \\
\text { Pers. }\end{array}$ & $\begin{array}{l}\text { Uniformly small round nodules occurring } \\
\text { all along the length of the firm roots; sur- } \\
\text { face smooth; nodule color white or light } \\
\text { pink; size } 1-1.5 \mathrm{~mm} \text {. }\end{array}$ & $\begin{array}{l}\text { A rare local plant, found in } \\
\text { silicaceous sand and moist } \\
\text { shale to sandy clay soils; } \\
\text { soil pH range from } 5 \text { to } 8 \text {. }\end{array}$ & $\begin{array}{l}\text { A sparse growing deep rooted } \\
\text { procumbent plant with } \\
\text { small slender leaves in } \\
\text { pairs; flowering and fruit- } \\
\text { ing year around. }\end{array}$ \\
\hline $\begin{array}{l}\text { Sub-family Caesalpin- } \\
\text { ioideae } \\
\text { Cassia mimosoides } \\
\text { Benth }\end{array}$ & $\begin{array}{l}\text { Fair number of nodules, borne on fine } \\
\text { branches mostly close to crown, delicately } \\
\text { attached and easily dislodged; young } \\
\text { nodules round, mature nodules long } \\
\text { jointed finger-like, some branching to } \\
\text { give glove-like appearance; surface } \\
\text { smooth; color distinctly brown; } 1-1.5 \mathrm{~mm} \\
\text { thick, and 4-5 mm long, occasionally } \\
\text { some very large nodules measured to } 1.0 \\
\text { cm in length. }\end{array}$ & $\begin{array}{l}\text { Cosmopolitan distribution in } \\
\text { dry to moist areas; near } \\
\text { neutral to alkaline soil (pH } \\
6.6-8.2 \text { ). }\end{array}$ & $\begin{array}{l}\text { Herbaceous, dense, slender } \\
\text { shrub, up to } 75 \mathrm{~cm} \text { tall, } \\
\text { usually gregareous; seeding } \\
\text { fair to heavy. }\end{array}$ \\
\hline
\end{tabular}

* Not previously recorded for nodulation. 


\section{EXPERIMENTAL PROCEDURE}

Field trips were taken periodically to different parts of the Island. The same sites were revisited at different times of the year. Normal healthy plants were collected and field observations were recorded regarding the plant, soil, and environmental conditions. The root system with associated nodules were dug out, placed in plastic bags and chilled in an ice-chest, where they remained until reaching the laboratory where the roots were washed and nodule characteristics noted and recorded.

Rhizobium bacteria were isolated after surface sterilization of the nodules with 0.1-percent zephiran chloride solution followed by inoculation of yeast-mannitol agar medium (1) containing asparagus extract with the crushed nodule sap as described earlier (6). Presence of gram-negative banded bacteria was taken as presumptive evidence of the Rhizobia isolates.

\section{RESULTS}

Among the indigenous tropical legumes surveyed, a total of 49 nodulated species were considered to have some potential value for agricultural use. Of these, 13 species had not been recorded for nodulation previously according to the global listing of nodulated and non-nodulated legumes maintained by Dr. O. N. Allen (personal communication). These were reported earlier (6) and are marked with an asterisk in table 1 which lists the 49 species and furnishes a description of the root nodule, plant habit, and habitat.

Contrary to the usual belief that nodulation occurs mostly in near-neutral to alkaline soils, the following well-nodulated native legumes were found to occur in acidic soils: Abrus praecatorius L., Aeschynomene portoricensis Urban, Calopogonium orthocarpum Urban, Dalbergia monetaria L.f., Desmodium adscendens (Sw.) DC., Desmodium barbatum (L.) Benth, Phaseolus trichocarpus C. Wright and Vigna vexillata (L.) A. Rich.

A few legumes were found in soils which varied in reaction from very acid to near-neutral, for example: Desmodium canum (gmel.) Sching \& Thellung, Vigna repens (L.) Kuntze., Zornia diphylla (L.) Pers.

These legumes may prove of real interest for cross-inoculation and legume-breeding investigations.

\section{SUMMARY}

A search was conducted in Puerto Rico for nodulated indigenous tropical legumes which show possible potential for agricultural purposes. Forty nine such legume species were encountered, of which 13 were new records for nodulation. Descriptions are given for the rhizobial nodules on the 49 species and agronomic observations on the growth habits of the plants and their habitats are furnished. 


\section{RESUMEN}

Se llevó a cabo en Puerto Rico una búsqueda por legumbres nativas tropicales noduladas de posible utilidad agrícola. Se hallaron 49 de estas especies de legumbres, de las cuales 13 eran noduladas y no se habian informado antes. Se describen los nódulos rizóbicos de las 49 especies y, además, se presentan observaciones agronómicas sobre el hábito de crecimiento de las plantas y sus habitats.

\section{LITERATURE CITED}

1. Allen, O.N., The isolation of rhizobia from root nodules, p. 69-70. In O. N. Allen (ed.) Experiments in soil bacteriology, Burgess Publishing Company, Minneapolis 15, Minn., 1953.

2. Bermudez Garcia, L. A., Indigenous legumes of the Cauca Valley (In Spanish), Rev. Acad. Colombiana Cienc. Exactas, Físicas y Natur. 11: 51-83, 1960.

3. Bryan, W. W., The search for tropical pasture legumes-a progress report, J. Australian Inst. Agr. Sci. 29: 149-53, 1963.

4. De Souza, D. I. A., Nodulation of indigenous Trinidad legumes, Trop. Agr. 43: 265-7, 1966.

5. Dirven, J. G. P., Some important grassland types in Surinam, Netherland J. Agr. Sci. 13 : 102-13, 1965.

6. Dubey, H. D., Woodbury, R., and Rodriguez, R. L., New records of tropical legume nodulation, Bot. Gaz. 133: 35-8, 1972.

7. Semple, A. T., Legumes in the pastures of Costa Rica, (in Spanish), Turrialba 12: $39-40,1962$. 